\title{
A Millimeter-Wave Scaled Measurement System for Wireless Channel Characterization
}

\author{
Farshid Aryanfar, Student Member, IEEE, and Kamal Sarabandi, Fellow, IEEE
}

\begin{abstract}
In this paper, a scaled measurement system designed for wireless channel characterization is proposed and discussed, and experimental results are reported. The system consists of a vector network analyzer, miniaturized $W$-band transmitter and receiver probes, scaled buildings, and a precise computer-controlled positioner. The scaled propagation measurement system is designed to operate coherently at the $90.8-92.8-G H z$ band, and it provides a dynamic range of $85 \mathrm{~dB}$. As the measurement wavelength in this system $(\cong 3 \mathrm{~mm})$ is approximately two orders of magnitude smaller than the actual wavelength in most commercial and military wireless systems, a typical dimension of the propagation environment can be smaller than the actual environment proportionally. Hence, propagation measurements can be done very efficiently under laboratory conditions using a scaled model of a city block. This system is intended for verifying the results of wave-propagation prediction software. It can also be used to investigate scattering models for different types of buildings and complex objects, data collection for wireless communication, and many other studies such as diversity methods (frequency and polarization).
\end{abstract}

Index Terms-Coplanar waveguide (CPW), millimeter wave, scaled model, subharmonic mixer, wave propagation, $W$-band.

\section{INTRODUCTION}

$\mathbf{T}$ HE RAPID evolution of wireless communications has lead to the use of higher frequency bands, smaller cell sizes, and smart antenna systems, making propagation prediction issues more challenging [1]. Accurate channel modeling in wireless communication allows for: 1) improved system performance (bit error rate, battery life, etc.) and 2) reduced interference, ensuring proper operation of other commercial systems and providing secure communication for military purposes.

Numerous methods and techniques have been developed to predict the effect of the channel. These can be divided to two categories, which are: 1) statistical or empirical models like Okumara et al. [2], Hata [3] and Longley and Rice [4] and 2) deterministic or analytical models like ray-tracing-based models [5]. Statistical models are based on measured data. Therefore, to develop these models, many measurement data sets are required. A drawback of these empirical models is that they are only applicable to environments similar to the measurement environment used for the model development. Therefore, to build a general and accurate model, an exorbitantly large number of measurement sets are required.

Manuscript received December 22, 2003; revised February 17, 2004. This work was supported by the U.S. Army Research Office.

The authors are with the Radiation Laboratory, Department of Electrical Engineering and Computer Science, The University of Michigan at Ann Arbor, Ann Arbor, MI 48109 USA (e-mail: faryanfa@eecs.umich.edu; saraband@eecs.umich.edu).

Digital Object Identifier 10.1109/TMTT.2004.828471
Deterministic methods are based on the physics of the environment and wave-propagation phenomena such as reflection, transmission, and diffraction. These methods are generally applicable to any arbitrary environment and are especially useful for microcellular and pico-cellular environments where statistical models fail as the channel is highly correlated to the environment. Propagation model verification using measured data is a very difficult task considering that all dimensions, coordinates, and dielectric properties of the scatterers in the environment must be measured and ported to the simulator code. Since these cannot be measured accurately, there remains a wide range of uncertainties in the simulation result.

An alternate approach to time-consuming and expensive outdoor measurements is the scaled measurement system proposed and discussed in this paper. This system allows accurate measurement of well-defined channels under a controlled laboratory environment. Scaled systems have been used to characterize performance of antennas on large platforms such as ships and airplanes [6]. However, the literature concerning scaled measurements for characterizing coherent wave propagation is scarce. In [7] and [8], a fixed scaled system, operating at $25 \mathrm{GHz}$, is reported for measuring wave transmission over simple two-dimensional (2-D) rectangular cylinders. In this paper, a sophisticated millimeter-wave scaled propagation measurement system (SPMS), the concept of which we presented in [9], is designed to measure all desired channel parameters over a realistic scaled urban environment. Confining the desired range of frequency to systems operating at UHF to the $L$-band $(0.5-2 \mathrm{GHz})$, dimensions of scatterers and terrain features in the scaled propagation channel can be reduced by a factor of 50-200 for the proposed SPMS that operates at around $100 \mathrm{GHz}$. This reduction brings the size of buildings from meters to centimeters so a scaled model of a city block can easily fit into a laboratory, and measurements can be done quickly, accurately, and cost effectively.

In what follows, the block diagrams and main components of the SPMS are first presented, and then the design, fabrication, and performance of individual elements will be described. The overall system specifications are demonstrated last.

\section{MiLlimeter-WaVE SPMS BLOCKS}

Fig. 1 shows the main components of the $W$-band SPMS. The system includes an $x-y-z$ probe positioner, scaled model of a city block, miniaturized $W$-band transmitter and receiver probes, and a vector network analyzer. The network analyzer in the SPMS is used for signal processing and data acquisition. Therefore, the setup is configured to characterize the propagation channel in a manner similar to the standard $S_{21}$ measure- 


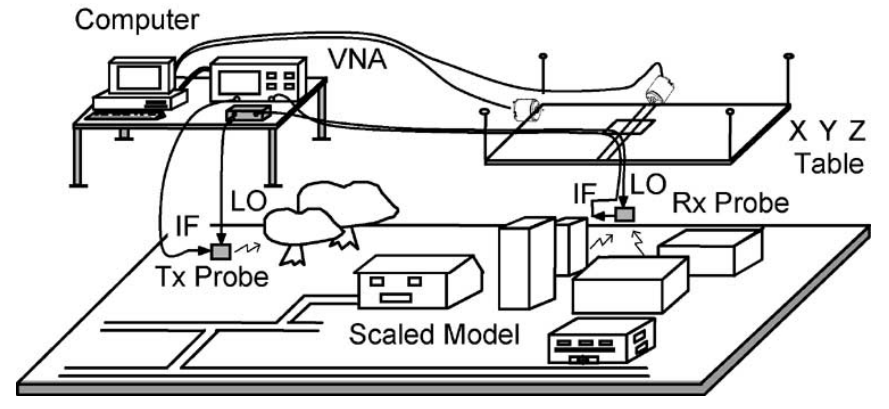

Fig. 1. SPMS block diagrams.

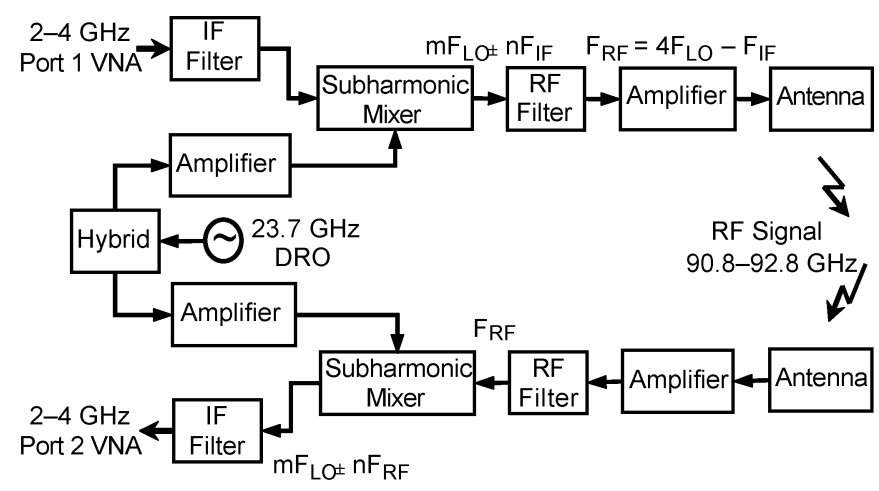

Fig. 2. $W$-band transmitter and receiver probes block diagrams.

ment. The network analyzer allows for coherent and broad-band path loss measurement with a wide dynamic range. The timedomain features of the network analyzer also allow for measuring the power delay profile, which makes the SPMS unique in channel modeling. In order to move the receiver probe with the required accuracy (to within a fraction of the wavelength $\cong 3 \mathrm{~mm}$ ) for measuring fast fading and slow fading statistics, a computer-controlled $x y$ table has been designed and built. As the operating frequency of the network analyzer ( $L$-band) is different from the required SPMS frequency ( $W$-band), an up-converter and down-converter has been designed and fabricated as part of the transmitter and receiver probes, respectively. To minimize the interaction of the probes with their environment, they must be designed as small as possible. Miniaturized transmitter and receiver probe designs are presented in the following sections.

\section{III. $W$-BAND TRANSCEIVERS}

As mentioned earlier, the signal from the network analyzer is up-converted and down-converted between the $W$ - and $L$-bands by the transmitter and receiver probes. Fig. 2 shows the block diagrams of the transmitter and receiver probes. As shown in the upper branch, the IF signal $\left(F_{\mathrm{IF}}\right)$ from the output of the network analyzer is mixed with the local-oscillator (LO) signal $\left(F_{\mathrm{LO}}\right)$ in a subharmonic mixer to generate the transmitter signal. This signal contains all harmonics of the form $m F_{\mathrm{LO}} \pm n F_{\mathrm{IF}}$. The desired harmonic, which results from mixing the fourth harmonic of the LO signal and IF signal, is selected by the RF filter for transmission. It is then amplified and transmitted. At the receiver, the RF signal captured by the antenna is amplified before down-conversion at the receiver subharmonic mixer. The desired IF signal $\left(4 F_{\mathrm{LO}}-F_{\mathrm{RF}}\right)$ is then selected by the IF filter and delivered to port 2 of the network analyzer after IF amplification (not shown). Subharmonic mixers are used to allow for stepped frequency operation without need for distributing a common $W$-band LO to mobile transmitter and receiver probes. The common $\mathrm{LO}$ at $23.7 \mathrm{GHz}$ for the subharmonic mixers is distributed by high-quality flexible coaxial cables. The use of a common LO allows for coherent measurement, as well as reduction of the network analyzer's IF bandwidth to its minimum value $(10 \mathrm{~Hz}$ for HP8720D). Narrow IF bandwidth reduces the noise level and permits measuring signals at very low power levels (around $-110 \mathrm{dBm}$ for HP8720D). The local signal source in the SPMS is generated by a dielectric-resonator oscillator that has a frequency variation of $6 \mathrm{kHz} /{ }^{\circ} \mathrm{C}$ and a phase noise of $-86 \mathrm{dBc} / \mathrm{Hz}$ at $10-\mathrm{kHz}$ offset from the center frequency that provides the required conditions.

The transceiver circuit was fabricated on a 10-mil $(\cong 250-\mu \mathrm{m})$-thick quartz wafer. As the width of a $50-\Omega$ microstrip line on available substrates becomes comparable with the wavelength at $W$-band frequencies, microstrip lines become inappropriate for circuit design. To be compatible with the test setup, the circuit was also designed and fabricated using coplanar waveguide (CPW) lines. The fabrication processes were performed in The University of Michigan at Ann Arbor's clean room, using the wet-etching technique on 3- $\mu$ m electroplated gold on the quartz wafer. The skin depth for the RF, LO, and IF frequencies are $0.26,0.52$, and $1.5 \mu \mathrm{m}$, respectively. The gold thickness is marginally sufficient for the IF signal, but as will be shown, the minimum feature size in the circuits is $10 \mu \mathrm{m}$, which limits the thickness of the plated gold that can be used. Fortunately, insufficient metal thickness does not degrade the circuit performance because, in this miniaturized circuit, the IF signal path on the circuit is just $2.5 \mathrm{~mm}$, which is smaller than $0.01 \lambda_{g}^{\mathrm{IF}}$. Therefore, the associated metallic loss is negligible.

The simulation results in the following sections were performed by ADS Momentum for the passive elements, and a harmonic-balance simulator for nonlinear analysis of the subharmonic mixer. The measurements were realized using a probe station (for on-wafer measurements), HP-8510C network analyzer, HP-W85104A millimeter-wave test setup, HP-8562A spectrum analyzer, and HP-11970W waveguide harmonic mixer.

\section{A. IF Filter}

The IF filter is placed to isolate the IF and RF signals in order to improve the subharmonic mixer's efficiency. There are many topologies that can be used for this filter. However, to minimize the size, a low-pass filter constructed from a quarter-wavelength high-impedance line terminated by an inter-digital capacitor is used. For this simple filter, the higher the capacitance and line impedance, the lower the RF signal leakage to the IF port. Hence, the aim is to increase the capacitance and the line impedance as much as possible. However, these two parameters are limited by the minimum achievable feature size in the fabrication process, which is approximately $10 \mu \mathrm{m}$. Fig. 3 shows the IF filter layout. For the specified dimensions in this figure, a line impedance of $145 \Omega$ and an interdigital capacitance of $75 \mathrm{fF}$ 


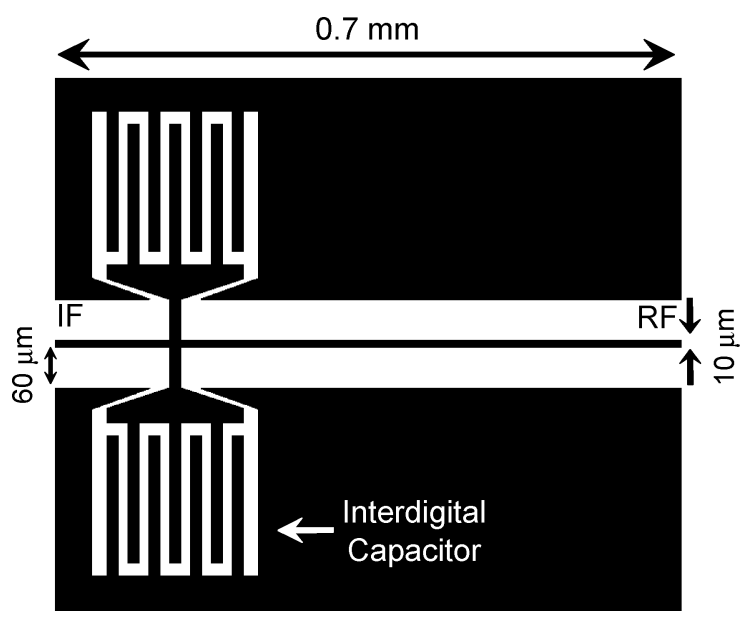

Fig. 3. IF filter layout and dimensions.

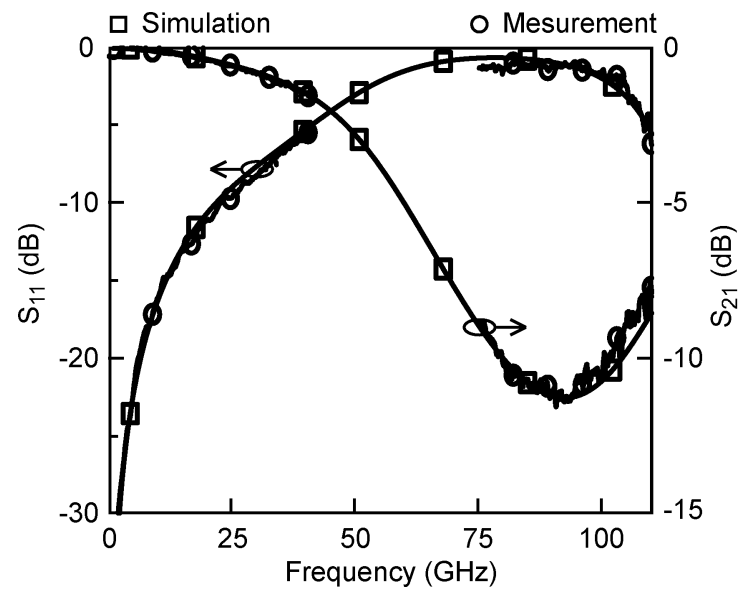

Fig. 4. Simulation and measurement results for IF filter.

with a quality factor of ten at the $W$-band are achieved. The method of moments (MoM) simulation and the measured transmission coefficient and return loss for the IF filter are plotted in Fig. 4, where excellent agreement is shown. There are no measured data between $40-75 \mathrm{GHz}$. The maximum insertion loss of this filter at the IF signal is less than $0.1 \mathrm{~dB}$, and its return loss is less than $-24 \mathrm{~dB}$ over the desired IF frequency range. The isolation between the RF and IF signals is more than $12 \mathrm{~dB}$.

\section{B. RF Filter}

The RF filter is intended for selecting the desired harmonic of the mixed IF and $\mathrm{LO}$ signals $\left(4 F_{\mathrm{LO}}-F_{\mathrm{IF}}\right)$ generated by the subharmonic mixer. It also prevents IF signal leakage to the RF port, which improves the conversion loss of the subharmonic mixer used for up-conversion and down-conversion. However, in the transmitter probe, in addition to RF-IF isolation, this filter should reject strong and undesired harmonics like the third and fifth harmonics of the LO to keep the RF amplifier from saturation. In order to achieve all of the above-mentioned features, the RF filter is made of two cascaded bandpass filters.

1) First Stage: A CPW coupled-line filter shown in Fig. 5 is selected as the first stage of the RF filter. The advantages of this filter are high isolation between the RF and IF signal, low insertion loss at the RF frequency range,

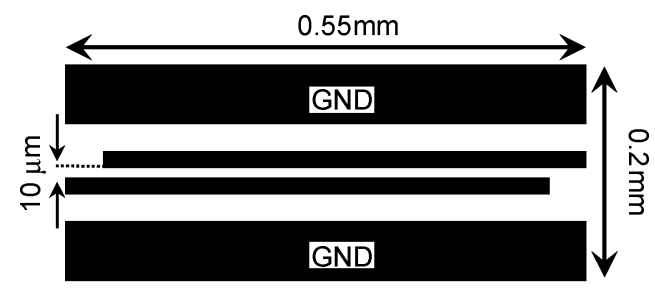

Fig. 5. CPW coupled line for the first stage of RF filter.

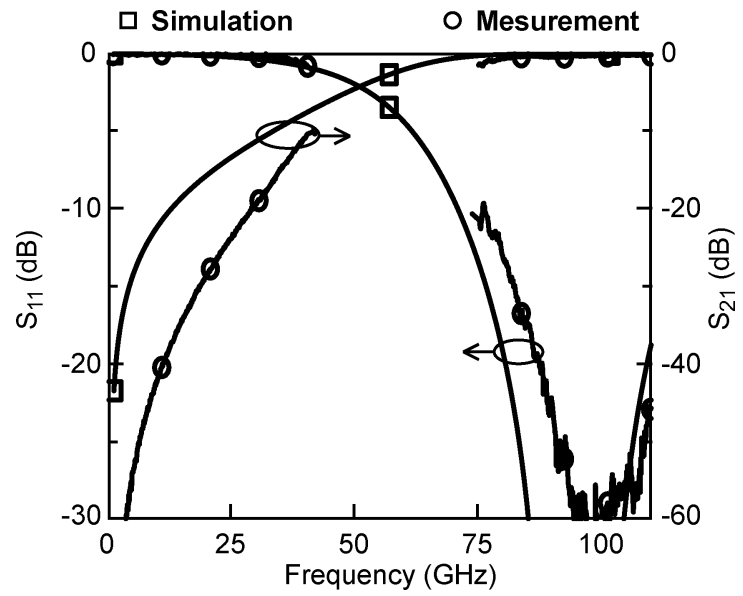

Fig. 6. Simulation and measurement results for the CPW coupled-line filter.

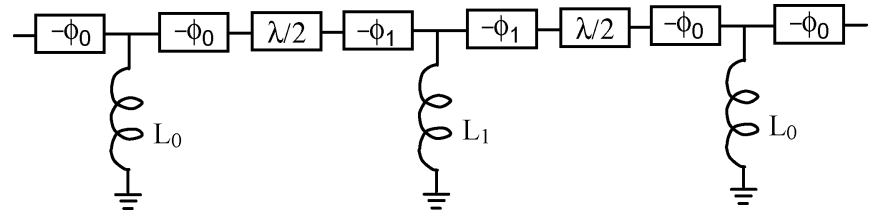

Fig. 7. Circuit model of inductive coupled resonator filter for the second stage of the RF filter.

compact size, and high impedance at the IF frequency. Fig. 6 shows the simulated and measured $S_{11}$ and $S_{21}$ of this filter as a function of frequency. As can be seen, this filter provides more than $50 \mathrm{~dB}$ of IF-to-RF isolation and has an insertion loss of less than $0.5 \mathrm{~dB}$ and a return loss of less than $-25 \mathrm{~dB}$ at the RF frequency range.

2) Second Stage: In order to generate a spurious-free RF signal and also prevent saturation of the RF amplifier by the undesired strong $\mathrm{LO}$ harmonics $\left(3 F_{\mathrm{LO}}\right.$ at $71.1 \mathrm{GHz}$ and $5 F_{\mathrm{LO}}$ at $118.5 \mathrm{GHz}$ ), created by the subharmonic mixer, a second stage of the RF filter is designed. The second stage is constructed from two-section inductively coupled resonators [10], [11], whose circuit model and topology are, respectively, shown in Figs. 7 and 8. The inductive coupling between the resonators is achieved by symmetric short-circuited CPW line stubs, as shown in Fig. 9(a). A simple method to calculate the inductance of these stubs is the classical formula for ribbon inductors [10]

$$
L=2 l\left\{\ln \left(\frac{2 \pi l}{w}\right)-1+\frac{w}{\pi l}\right\} \mathrm{nH}
$$

where $w$ and $l$ (in centimeters), are the width and length of the inductor, respectively. However, the accuracy of 


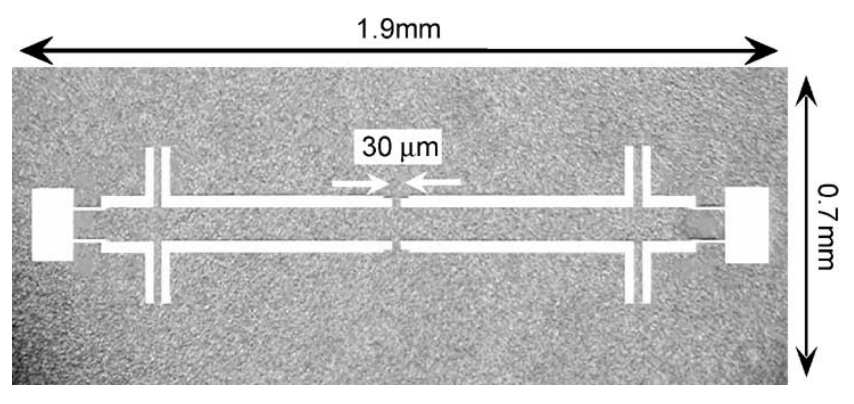

Fig. 8. Fabricated inductive coupled resonator filter on quartz wafer.

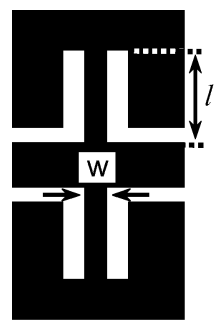

(a)

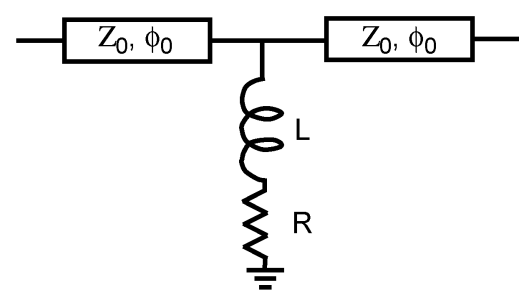

(b)
Fig. 9. Characterization of effective inductance and resistance for short stubs in CPW line. (a) Inductor layout. (b) Circuit model.

TABLE I

EFFECTIVE INDUCTANCE OF SHORT STUBS IN CPW LiNE

\begin{tabular}{ccccc}
\hline Inductor\# & $w(\mu m)$ & $l(\mu m)$ & $\mathrm{MoM}(\mathrm{pH})$ & Eq. $1(\mathrm{pH})$ \\
\hline 1 & 60 & 20 & 5.1 & 1.4 \\
\hline 2 & 30 & 20 & 7.1 & 1.8 \\
\hline 3 & 30 & 136 & 21.1 & 32.9 \\
\hline 4 & 30 & 198 & 25.0 & 54.9 \\
\hline 5 & 25 & 213 & 33.0 & 64.3 \\
\hline
\end{tabular}

this formula is quite poor with errors often greater than $100 \%$. Therefore, to extract an accurate effective inductance of these short stubs, the MoM simulated $S$-parameters of the stubs, shown in Fig. 9(a), are compared with its circuit model [shown in Fig. 9(b)]. Table I shows the calculated inductances using (1) and the extracted values from the MoM simulation. The MoM results are used in the final design and, as will be shown, they lead to excellent agreement between the measured and simulated filter responses.

In order to provide the required out-of-band rejection and minimum insertion loss simultaneously, a two-pole filter is found to be the optimum choice. The design of this filter began with the corresponding low-pass element values $g_{0} \ldots g_{n}$. Using (2)-(4), $L_{j}\left(X_{j} / \omega_{0}\right)$ and $\phi_{j}$ are then calculated [10] as follows:

$$
\frac{Z_{0}}{X_{j}}=\left\{\begin{array}{l}
\left(\frac{Z_{0}}{S}\right)^{\frac{1}{2}}-\left(\frac{S}{Z_{0}}\right)^{\frac{1}{2}}, \quad j=1, n+1 \\
\frac{Z_{0 \sqrt{g_{j-1} g_{j}}}}{S g_{0} g_{1}}-\frac{S \quad g_{0} g_{1}}{Z_{0 \sqrt{g_{j-1} g_{j}}}}, \quad j=2,3, \ldots, n
\end{array}\right.
$$

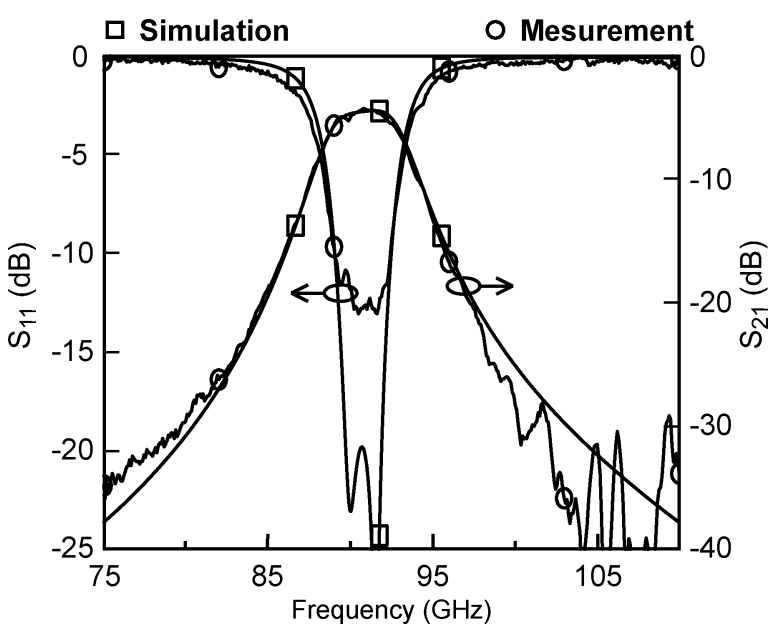

Fig. 10. Simulation and measurement results for the inductive coupled resonator filter.

$$
\begin{aligned}
S & =\frac{\pi Z_{T}}{2 g_{o} g_{1}} \frac{\omega_{2}-\omega_{1}}{\omega_{0}} \\
\phi_{j} & =\tan ^{-1} \frac{2 X_{j}}{Z_{0}}
\end{aligned}
$$

where $Z_{T}$ and $Z_{0}$ are the characteristic impedance of the CPW line and port impedances, respectively. In this design, both are chosen to be $50 \Omega$. In (3), $\omega_{0}, \omega_{1}$, and $\omega_{2}$ are the center, lower cutoff, and higher cutoff angular frequencies. A photograph of the fabricated filter is shown in Fig. 8. Fig. 10 shows the simulated and measured filter responses. The magnetic current concept is used in the MoM simulation for fast computation and more accurate excitation of CPW structures. As such, conductive loss is not modeled. This effect was considered in simulation by extracting inductors and CPW line parameters from measured results and was used in the simulations. Fig. 10 shows the filter rejection at $3 F_{\mathrm{LO}}$ and $5 F_{\mathrm{LO}}$ to be more than $35 \mathrm{~dB}$. The closest undesired harmonic to the RF signal, which is the upper sideband of the up-converted IF signal $\left(4 F_{\mathrm{LO}}+F_{\mathrm{IF}}=\right.$ $96.8-98.8 \mathrm{GHz}$ ), is also at least $30 \mathrm{~dB}$ attenuated through two such filters at the transmitter and receiver probes totally. This ensures that the SPMS is able to measure fading depth at least as low as $30 \mathrm{~dB}$.

\section{Subharmonic Mixer}

The conversion loss and noise performance of a millimeter-wave mixer usually is limited by insufficient LO power or by excessive LO noise [12]. Generally mixers are pumped at a half or quarter of the required LO frequency. The major disadvantage of this technique is a higher conversion loss compared to fundamental mixers. Considering the transmitter probe's block diagrams, the extra conversion loss of the subharmonic mixer is tolerable as long as the up-converted signal power reaches to the minimum input power to achieve maximum distortion-free output power of the RF amplifier, which, in this case, is $-24 \mathrm{dBm}$.

An antiparallel diode pair is a common choice for subharmonic mixers [12]. The reason is the symmetrical $V-I$ characteristic of the antiparallel diodes that suppresses the fundamental and even harmonics mixing product of the LO and RF (or IF) 
TABLE II

GaAs SCHOTTKY DIODES CHARACTERISTICS

\begin{tabular}{ccccc}
\hline$R_{s}(\Omega)$ & $R_{j}(\Omega)$ & $C_{j o}(\mathrm{fF})$ & $C_{T}(\mathrm{fF})$ & $V_{B R}(\mathrm{~V})$ \\
\hline 4 & 2.6 & 20 & 45 & 7.0 \\
\hline
\end{tabular}

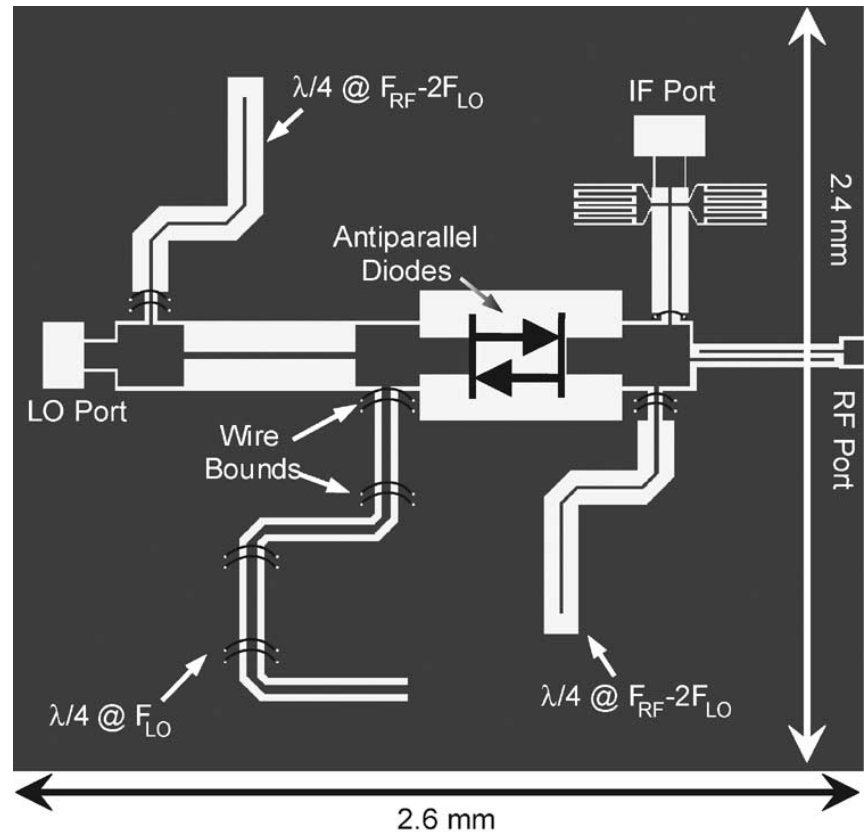

Fig. 11. Subharmonic mixer layout with IF and part of RF filters.

signal. It should be noted that proper operation of the subharmonic mixer depends on the similarity of the two back-to-back diodes. In our design, we have used a GaAs flip-chip Schottky antiparallel diode pair manufactured by MACOM, Lowell, MA. The specifications of these diodes are given in Table II.

In order to improve the conversion loss of the mixer, the mixing product near the second harmonic of the LO signal must be reactively terminated. Therefore, two quarter-wavelength open stubs centered at $F_{0}=F_{\mathrm{RF}}-2 F_{\mathrm{LO}}$ are placed at both sides of the antiparallel diodes to suppress the associated harmonics with the second harmonic of the LO signal. As mentioned earlier, the RF and IF filters prevent IF and RF signal leakage to the RF and IF ports, respectively. A quarter-wavelength short stub at the LO frequency, which acts as an open circuit for the LO signal and a short circuit for the IF and RF $\left(F_{\mathrm{RF}} \cong 4 F_{\mathrm{LO}}\right)$ signals, is also placed at the LO side of the subharmonic mixer to block IF and RF signals leakage to the LO port. The subharmonic mixer circuit is optimized for the best conversion loss, large signal matching at all ports, and minimum size. Fig. 11 shows the subharmonic mixer layout with the IF and the first stage of the RF filters. Wire bonds are placed at all discontinuities to suppress undesired slot modes on the CPW line.

The simulation and measured output RF power of the up-converter and conversion loss are shown in Figs. 12 and 13, respectively. As can be seen, the maximum up-converted signal power is sufficient to provide the RF amplifier with the required input power for maximum output. The maximum spurious level of the

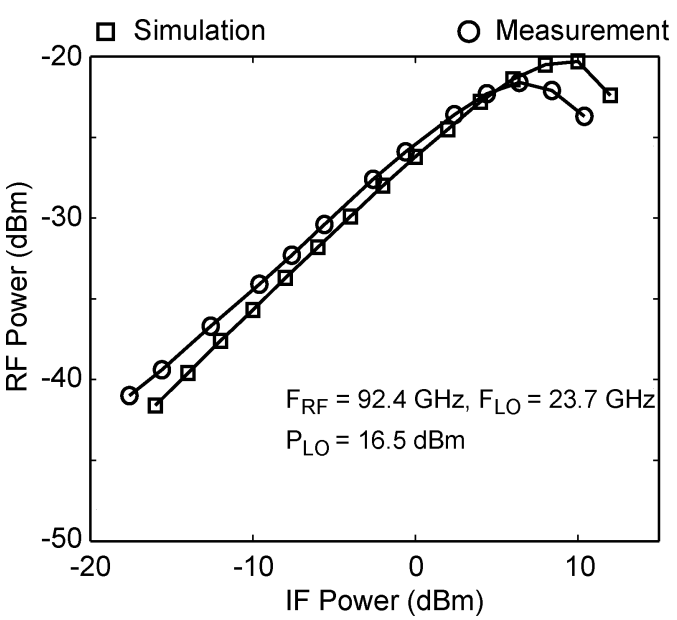

Fig. 12. Simulated and measured RF power at the up-converter output.

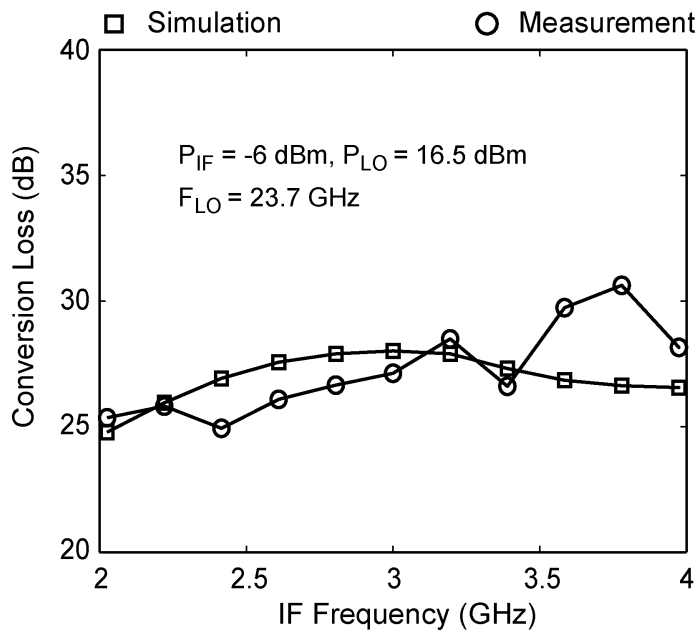

Fig. 13. Simulated and measured conversion loss of the up-converter.

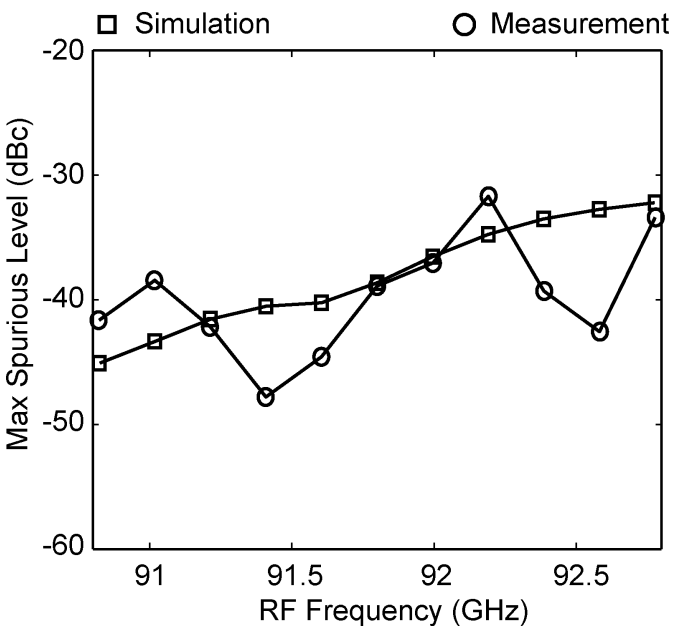

Fig. 14. Simulated and measured spurious level of the RF signal in SPMS.

RF signal in the SMPS is shown in Fig. 14, where it is shown that the average maximum spurious level is $-40 \mathrm{dBc}$. This allows for measurement of fading depths as low as $40 \mathrm{~dB}$. The down-converter used in the receiver probe has the same topology as the up-converter with similar performance characteristics. 


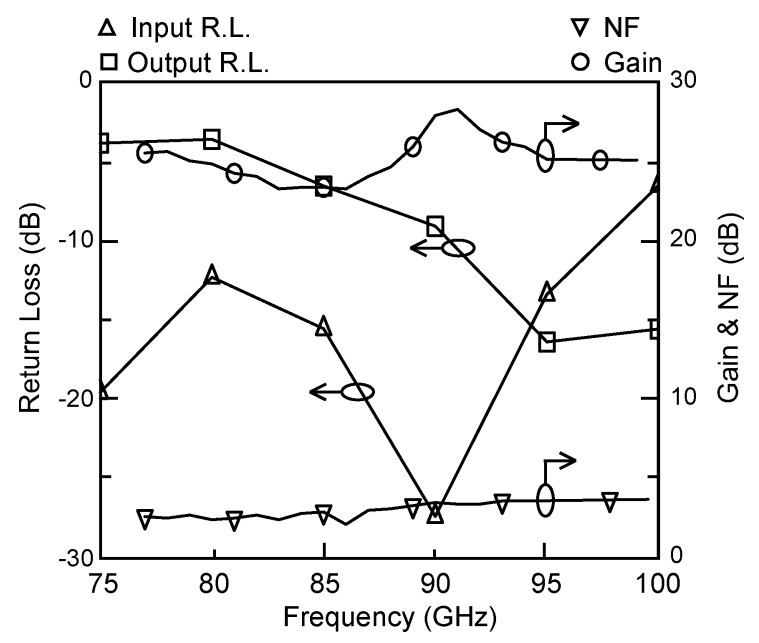

Fig. 15. RF amplifier gain, noise figure, and input and output return losses.

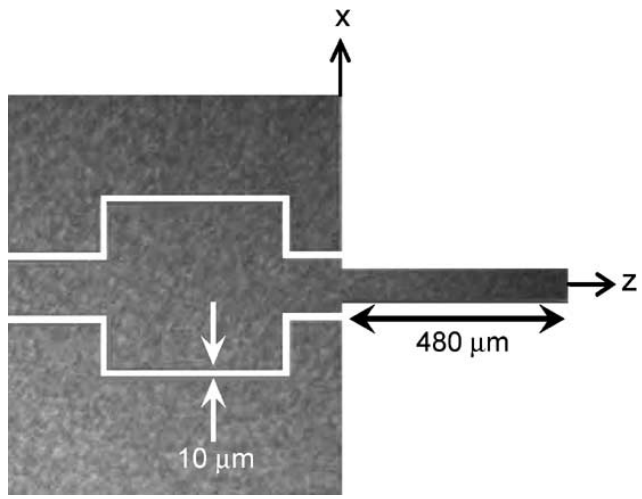

Fig. 16. Monopole antenna and matching line.

\section{RF Amplifier}

In order to compensate for the conversion losses of the up-converter and down-converter, a $W$-band amplifier is used in each probe. The amplifier chip is mounted on the circuit using silver epoxy. The input and output of the chip and dc contacts are connected to the circuit using gold wire bonds. In the desired RF frequency range, the amplifier has a gain of $27-29 \mathrm{~dB}$ and a noise figure of $4 \mathrm{~dB}$. Fig. 15 shows the amplifier gain, noise figure, and its input and output return losses.

\section{E. Antennas}

The main goal of the millimeter-wave scaled measurement system is to characterize propagation channels under laboratory conditions. In order to accomplish this properly, the transmit and receive antennas should have broad beam patterns. A monopole antenna is chosen for this purpose. As the monopole above a finite ground surface of the package is not automatically matched, a quarter-wavelength transmission line is used to match the antenna to the circuit. Fig. 16 shows the antenna and matching line between the antenna and RF amplifier. The simulated gain patterns of this antenna, above the packaged circuit, at $E$ - and $H$-planes are shown in Fig. 17(a) and (b), respectively.

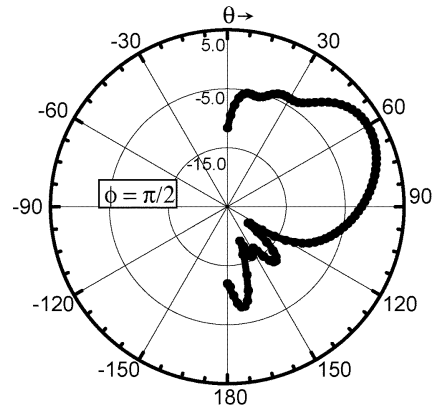

(a)

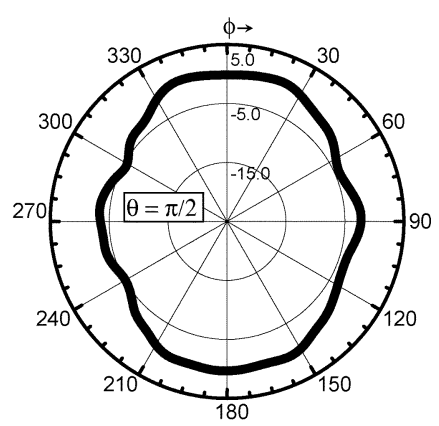

(b)
Fig. 17. Simulated gain pattern of monopole antenna above the packaged circuit. (a) $E$-plane. (b) $H$-plane.

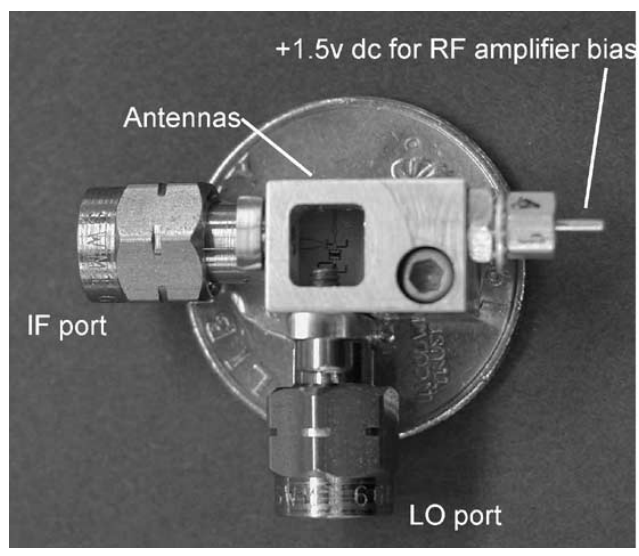

Fig. 18. Packaged RF probe against a quarter.

\section{F. Packaging}

The required accuracy in package dimensions has to be of the same order of the circuit elements that are connected to the package. For example, in a $W$-band system, an error as small as $10 \mu \mathrm{m}$ in the antenna's position can change its resonant frequency by approximately $2 \mathrm{GHz}$ and cause mismatching. A metallic package is designed using AutoCAD. In order to achieve the desired accuracy, the package was milled at The University of Michigan at Ann Arbor's space research machine shop, using a high precision HAAS, Tool Room Model CNC machine with tolerances less than $2.5 \mu \mathrm{m}$. The fabricated circuit on the quartz substrate was diced using an automatic dicing saw and then, together with 2.4-mm coaxial connectors for the IF and LO ports, was assembled with the aluminum package. The LO and IF 2.4-mm connector pins are connected to the circuit using silver epoxy. Fig. 18 shows the packaged probe against a quarter.

\section{SCALED BuILDINGS MOdELS}

As mentioned earlier, SPMS is designed to evaluate the performance of physics-based propagation models. As such, beside electronic precision for signal amplitude and phase measurement over a wide dynamic range, accurate rendition of the environment is also important. This includes accurate knowledge of geometrical features of scatterers (like buildings), as well as their material properties. To accommodate these features, scaled 


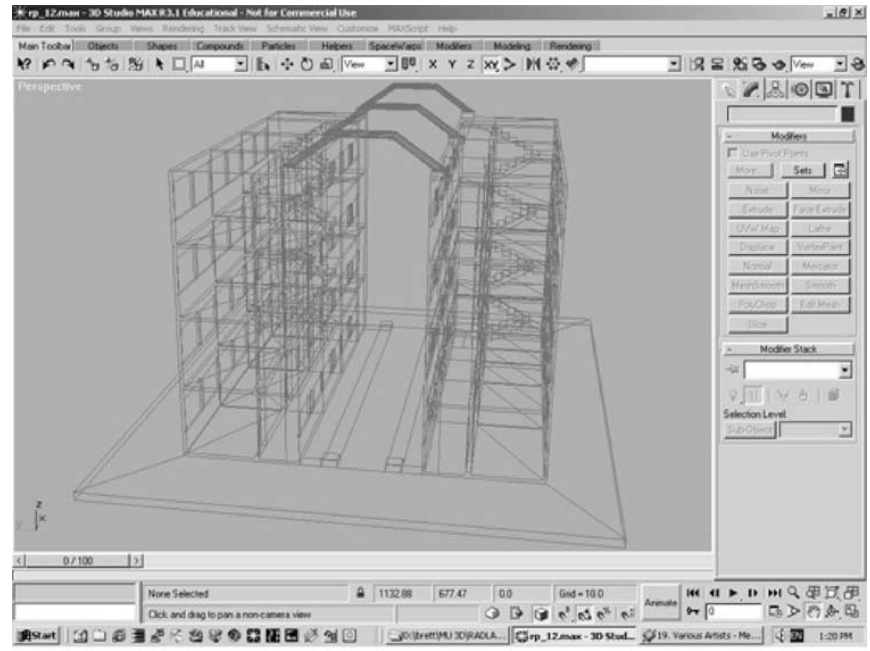

(a)

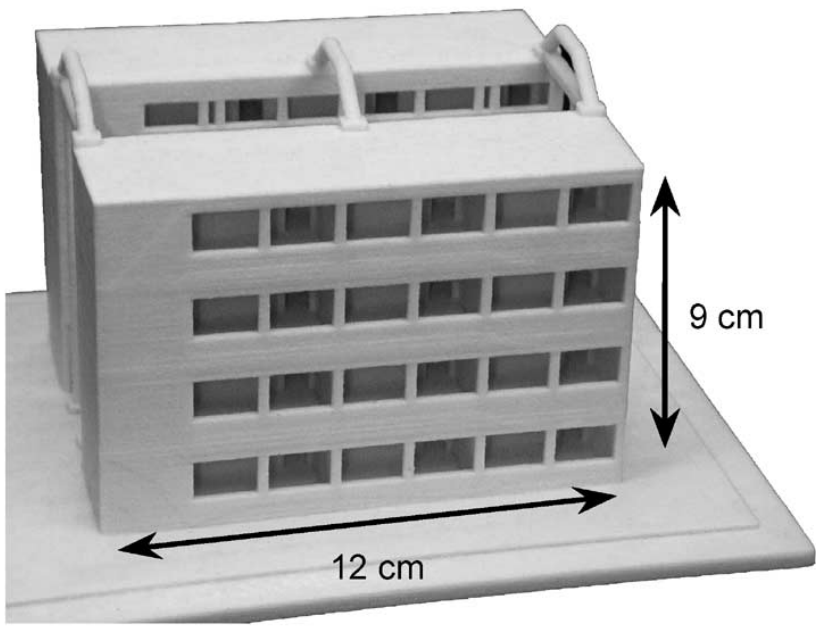

(b)

Fig. 19. Scaled building. (a) CAD model. (b) Printed building.

buildings and other scatterers with an arbitrary degree of complexity and well-characterized dielectric properties are used.

A precise three-dimensional (3-D) printer is used to make scaled buildings. This printer can use different materials and can make any building with any desired fine features. Any standard computer-aided design (CAD) software can be used to draw the buildings and export the geometry file for the 3-D printer. Fig.19 (a) and (b) shows the CAD model of a scaled building and actual building printed by the 3-D printer. Fig. 20 shows the first version of a scaled city block with simple building structures. It can be seen that the scaled city has a flexible grid, which is designed to help make an arbitrary arrangement of the blocks including roads, sidewalks, cars, and buildings.

\section{DieleCtric MEASUREMENT}

Dielectric properties of scatterers are needed for numerical simulation of wave propagation. Hence, the material used to make the blocks must be characterized at $W$-band frequencies. In this study, different techniques are used to characterize the real and imaginary parts of the dielectric constant of the material used in constructing the scaled buildings over a wide range of frequency. The first method is based on capacitor measurements

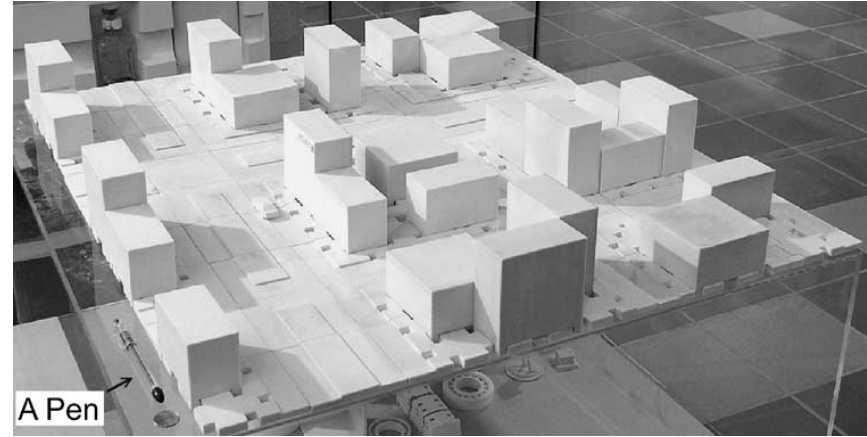

Fig. 20. Scaled city block.

TABLE III

MEASUREd EFFECTIVE Dielectric CONSTANT

\begin{tabular}{cccc}
\hline Frequency Band & $\mathrm{L}$ & $\mathrm{X}$ & $\mathrm{W}$ \\
\hline Sample 1 & $2.7-\mathrm{j} 0.05$ & $2.40-\mathrm{j} 0.04$ & $2.34-\mathrm{j} 0.03$ \\
\hline Sample 2 & $3.05-\mathrm{j} 0.15$ & $2.70-\mathrm{j} 0.07$ & $2.48-\mathrm{j} 0.06$ \\
\hline
\end{tabular}

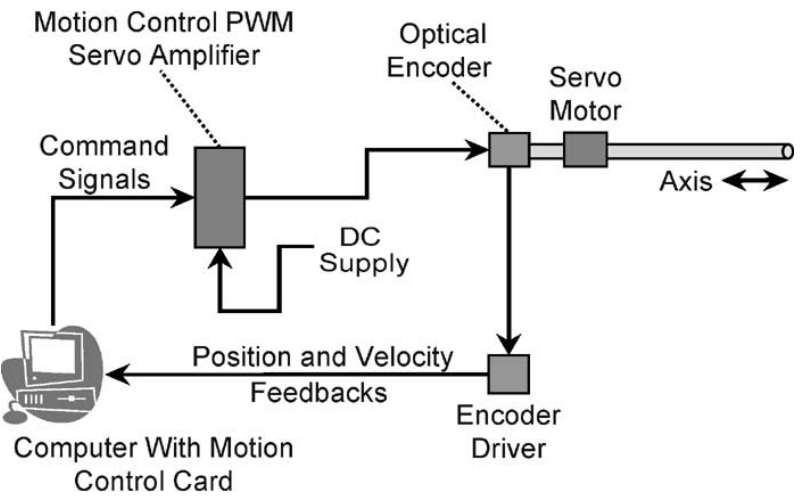

Fig. 21. $x-y$ table block diagrams.

at the $L$-band and below. ${ }^{1}$ The second method uses transmission and reflection measurements in a WR-90 $X$-band waveguide [13]. The third dielectric measurement is done at the $W$-band using transmission measurement through a dielectric slab at different incidence angles, and reflection measurement of the back metal dielectric slab [14]. The lower frequency dielectric measurements are mainly done to verify the measured results at the $W$-band. The measurement results for two different samples are shown in Table III. The permittivity of material used in construction of the scaled buildings resembles those of bricks and concrete.

\section{VI. $x-y$ TABLE}

A computer-controlled $x-y$ table that places the receiver probe at any arbitrary position within a $1.5 \mathrm{~m} \times 1.5 \mathrm{~m}$ area was designed and built. The system includes a motion control card, two step motors, power amplifiers, an encoder, and drivers. The computer issues commands to the motion control card, which, in turn, triggers the power amplifier to drive the motor. An optical encoder attached to each motor sends the position and velocity data back to the computer. The computer uses this information to control the probe movement. The system placement is accurate within

\footnotetext{
${ }^{1} \mathrm{HP}$ 85070M Dielectric Probe Measurement System, Agilent Technol., Palo
} Alto, CA, 1998. 
$0.25 \mathrm{~mm}$. This is acceptable accuracy even for fast fading measurements at the RF frequency range $(90.8-92.8 \mathrm{GHz})$ at which the wavelength is approximately $3.3 \mathrm{~mm}$. Fig. 21 shows the block diagram of the $x-y$ table system.

\section{CONCLUSION}

The design and fabrication of a $W$-band coherent transmission measurement system for measuring characteristics of wireless channels under laboratory conditions has been presented. The signal frequency of the transmission system is chosen to be much higher than most commercial and military wireless systems so that the size of the scatterers can be made sufficiently small. Frequency scaling from the $L$ - to the $W$-band allows for size reduction (scaling) of a city block down to two orders of magnitude. First, the overall system concept was introduced and the specifications of each system block were determined. Each system block was then fabricated, tested, and incorporated into the transmitter and receiver probes of the scaled measurement system. The proposed system offers unique capabilities including polarimetric and coherent path loss measurement within a very large dynamic range, accurate determination of fast and slow fading statistics, and characterization of the channel time-delay profile. These system capabilities are envisioned for verifying the accuracy of wave existing propagation channel simulators, developing scattering models and macro-models for different types of buildings and complex objects used for physics-based wave-propagation simulators, and data collection for wireless communication.

\section{REFERENCES}

[1] M. F. Iskandar and Z. Yun, "Propagation prediction models for wireless communication systems," IEEE Trans. Microwave Theory Tech., vol. 50, pp. 662-673, Mar. 2002.

[2] Y. Okumara, E. Ohmori, T. Kawano, and K. Fukuda, "Field strength variability in VHF and UHF land mobile service," Rev. Elect. Commun. Lab., vol. 16, no. 9-10, pp. 825-873, Sept.-Oct. 1968.

[3] M. Hata, "Empirical formula for propagation loss in land mobile radio services," IEEE Trans. Veh. Technol., vol. VT-29, pp. 317-325, Aug. 1980.

[4] A. G. Longley and P. L. Rice, "Prediction of tropospheric radio transmission loss over irregular terrain-a computer method," Inst. Telecommun. Sci., Environmental Sci., Boulder, CO, Tech. Rep. ERL 79-ITS 67, vol. 16, July 1968.

[5] F. Aryanfar and S. Safavi-Naeini, "Electromagnetic modeling of radio wave propagation in micro- and pico-cellular environments," in IEEE Antennas and Propagation for Wireless Communications Conf., vol. 43, Nov. 1998, pp. 25-28.

[6] J. H. Schukantz, D. W. S. Tam, J. B. McGee, L. B. Koyama, J. W. Rockway, and S. T. Li, "Transient scattering response of an airborne target," in Electromagnetic Compatibility Symp., Aug. 1995, pp. $620-623$.

[7] D. Erricolo, G. D'Elia, and P. L. E. Uslenghi, "Measurements on scaled models of urban environments and comparisons with ray-tracing propagation simulation," IEEE Trans. Antennas Propagat., vol. 50, pp. 727-735, May 2002.

[8] D. Erricolo, U. G. Crovella, and P. L. E. Uslenghi, "Time-domain analysis of measurements on scaled urban models with comparisons to raytracing propagation simulation," IEEE Trans. Antennas Propagat., vol. 50, pp. 736-741, May 2002.

[9] F. Aryanfar and K. Sarabandi, "Evaluation of a wave propagation simulator using a $95 \mathrm{GHz}$ transceiver system," in IEEE AP-S Int. Symp., vol. 4, June 2003, pp. 900-902.

[10] J. K. A. Everard and K. K. M. Cheng, "High performance direct coupled bandpass filters on coplanar waveguide," IEEE Trans. Microwave Theory Tech., vol. 41, pp. 1568-1571, Sept. 1993.

[11] A. Vogt and W. Jutzi, "An HTS narrow bandwidth coplanar shunt inductively coupled microwave bandpass filter on $\mathrm{LaAlO}_{3}$," IEEE Trans. Microwave Theory Tech., vol. 45, pp. 492-497, Apr. 1997.
[12] S. A.Stephen A. Maas, Microwave Mixers. Boston, MA: Artech House, 2003

[13] K. Sarabandi and F. T. Ulaby, "Technique for measuring the dielectric constant of thin materials," IEEE Trans. Instrum. Meas., vol. 37, pp. 631-636, Dec. 1988.

[14] F. T. Ulaby, R. K. Moore, and A. K. Fung, Microwave Remote Sensing Active and Passive. Norwood, MA: Artech House, 1982.

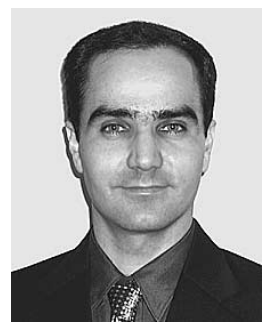

Farshid Aryanfar (S'01) was born in Mashad, Iran, in 1972. He received the B.S. degree in electrical engineering and M.S. degree with an emphasis on electromagnetics from the University of Tehran, Tehran, Iran, in 1994 and 1998, respectively, and is currently working toward the Ph.D. degree in electrical engineering and computer science with an emphasis on applied electromagnetics and RF circuits at The University of Michigan at Ann Arbor. His M.S. thesis concerned wave-propagation modeling in microcellular and pico-cellular environment using the ray-tracing algorithm, which later led to a 3-D physics-based wave-propagation simulator for wireless channel characterization.

From 1997 to 2000, he was Senior Microwave Engineer with the Informatics Services Corporation (ISC), where he was involved in developing satellite terminals and nondirectional beacon (NDB) transmitters. His current research interests include microwave/millimeter-wave circuits and systems, wave propagation in complex environment, and wireless channel characterization.

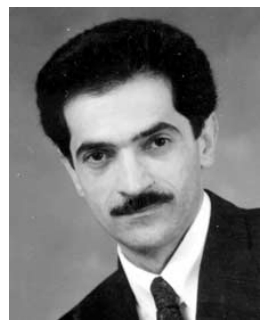

Kamal Sarabandi (S'87-M'90-SM'92-F'00) received the B.S. degree in electrical engineering from the Sharif University of Technology, Tehran, Iran, in 1980, and the M.S.E. and Ph.D. degrees from The University of Michigan at Ann Arbor, in 1986 and 1989, respectively, both in electrical engineering.

He is currently the Director of the Radiation Laboratory and a Professor with the Department of Electrical Engineering and Computer Science, The University of Michigan at Ann Arbor. His research areas of interest include microwave and millimeter-wave radar remote sensing, electromagnetic-wave propagation, and antenna miniaturization. He possesses 20 years of experience with wave propagation in random media, communication channel modeling, microwave sensors, and radar systems and is leading a large research group including four research scientists, 12 Ph.D. and two M.S. students. Over the past ten years, he has generated 20 Ph.D. students. He was the Principal Investigator of many projects sponsored by the National Aeronautics and Space Administration (NASA), Jet Propulsion Laboratory (JPL), Army Research Office (ARO), Office of Naval Research (ONR), Army Research Laboratory (ARL), National Science Foundation (NSF), Defense Advanced Research Projects Agency (DARPA), and numerous industries. He has authored numerous book chapters and over 115 papers appearing in refereed journals on electromagnetic scattering, random media modeling, wave propagation, antennas, microwave-measurement techniques, radar calibration, inversescattering problems, and microwave sensors. He has also authored or coauthored over 230 papers and invited presentations in many national and international conferences and symposia on similar subjects. He is listed in Who's Who in American Men and Women of Science, Who's Who in America, and Who's Who in Electromagnetics.

Dr. Sarabandi is a vice president of the IEEE Geoscience and Remote Sensing Society (GRSS), chairman of the Awards Committee of the IEEE GRSS, and a member of the IEEE Technical Activities Board Awards Committee. He is the associate editor of the IEEE TRANSACTIONS ON ANTENNAS AND PROPAGATION and the IEEE SENSORS JOURNAL. He is also a member of Commission F of URSI and The Electromagnetic Academy. He was the recipient of the Henry Russel Award from the Regent of The University of Michigan (the highest honor the University of Michigan bestows on a faculty member at the assistant or associate level), the 1999 GAAC Distinguished Lecturer Award presented by the German Federal Ministry for Education, Science, and Technology, which is given to approximately ten individuals worldwide in all areas of engineering, science, medicine, and law, and a 1996 Teaching Excellence Award presented by the Electrical Engineering and Computer Science Department, The University of Michigan at Ann Arbor. Over the past several years, joint papers presented by his students at a number of symposia [IEEE Antennas and Propagation Society (IEEE AP-S) Symposia (1995, 1997, 2000, and 2001); the IEEE International Geoscience and Remote Sensing Symposium (1999); and the IEEE Microwave Theory and Techniques Society (IEEE MTT-S) International Microwave Symposium (IMS) (2001)] have been the recipients of Student Prize Paper Awards. 